\title{
Rapid quantitation of 25-hydroxyvitamin D2 and D3 in human serum using liquid chromatography/drift tube ion mobility-mass spectrometry
}

Nicholas R. Oranzi ${ }^{1}$, Jiajun Lei ${ }^{1}$, Robin H. J. Kemperman ${ }^{1}$, Christopher D. Chouinard², Brett Holmquist ${ }^{3}$, Timothy J. Garrett ${ }^{4}$, Richard A. Yost ${ }^{1,4}$

${ }^{1}$ University of Florida, Department of Chemistry, Gainesville, FL

${ }^{2}$ Florida Institute of Technology, Department of Biomedical and Chemical Engineering,

Melbourne, FL

3LabCorp, Burlington, NC

${ }^{4}$ University of Florida, Department of Pathology, Gainesville, FL

\section{Contents:}

S-2. calibration curves for 25-hydroxyvitamin D2 and D3

S-2. Chromatogram after drift-time filtering

S-3. Correlation plot of patient samples quantified by vendor software and Skyline

S-3. Relative difference plots for vendor software and Skyline

S-4. Sample preprocessing in MassHunter

S-5. Relative informing power of IM-HRMS

S-6. References 


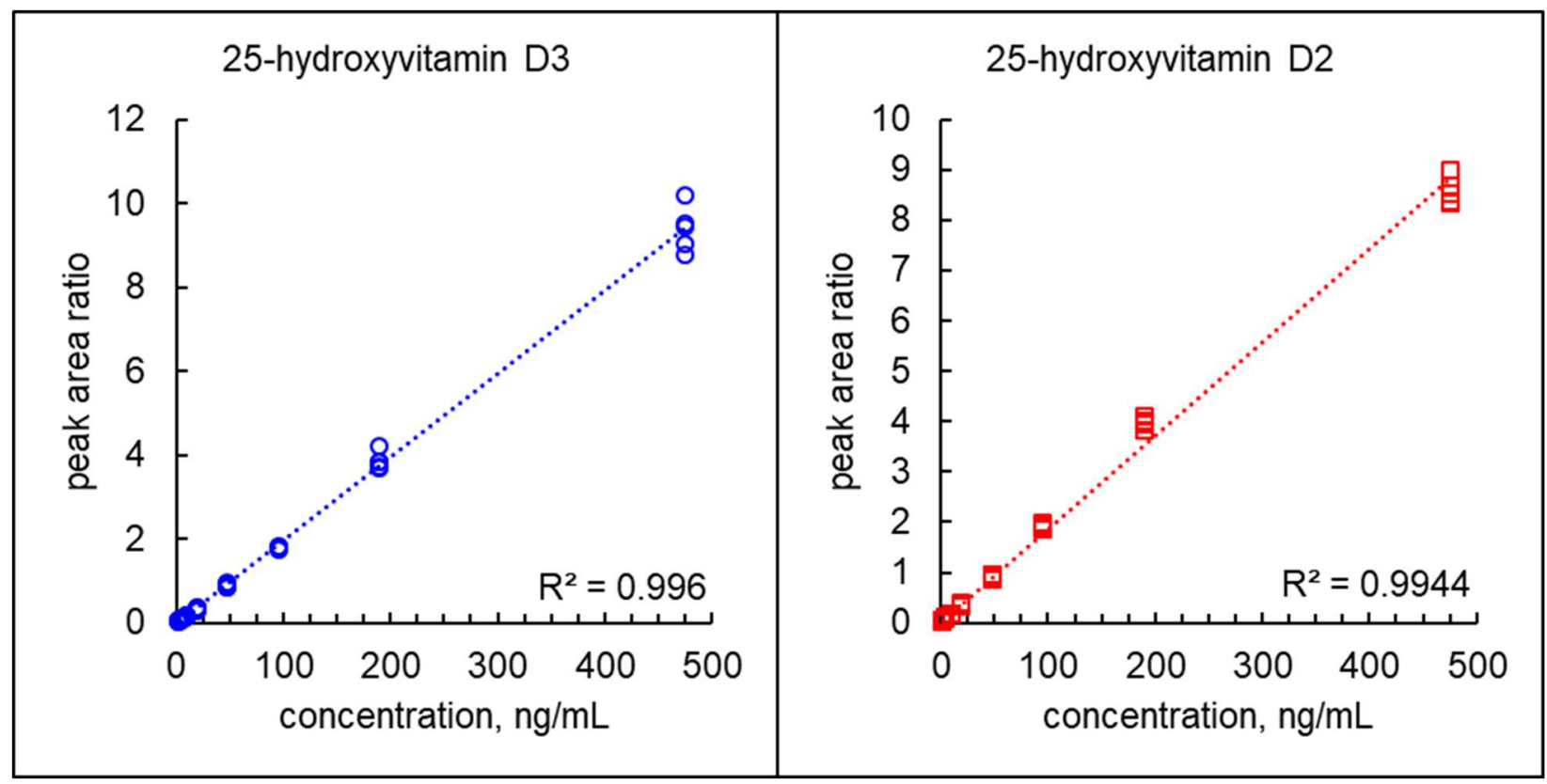

Figure S1. Eight-point calibration curves ( $n=5 /$ level) showing linearity between 2 and $500 \mathrm{ng} / \mathrm{mL}$ for $25 \mathrm{OHD} 2$ and $25 \mathrm{OHD} 3$

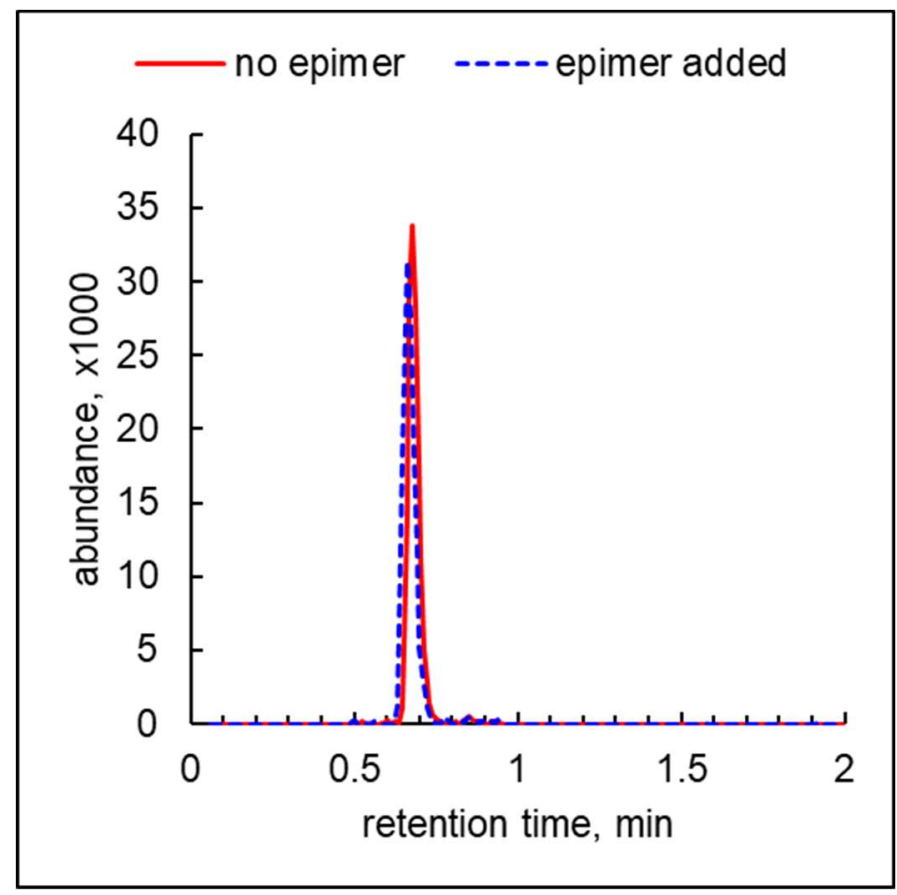

Figure S2. Extracted drift time and ion chromatogram for $75 \mathrm{ng} / \mathrm{mL}$ quality control with and without $50 \mathrm{ng} / \mathrm{mL}$ epi25OHD3. Drift time filtering removed interference from the epimer. 


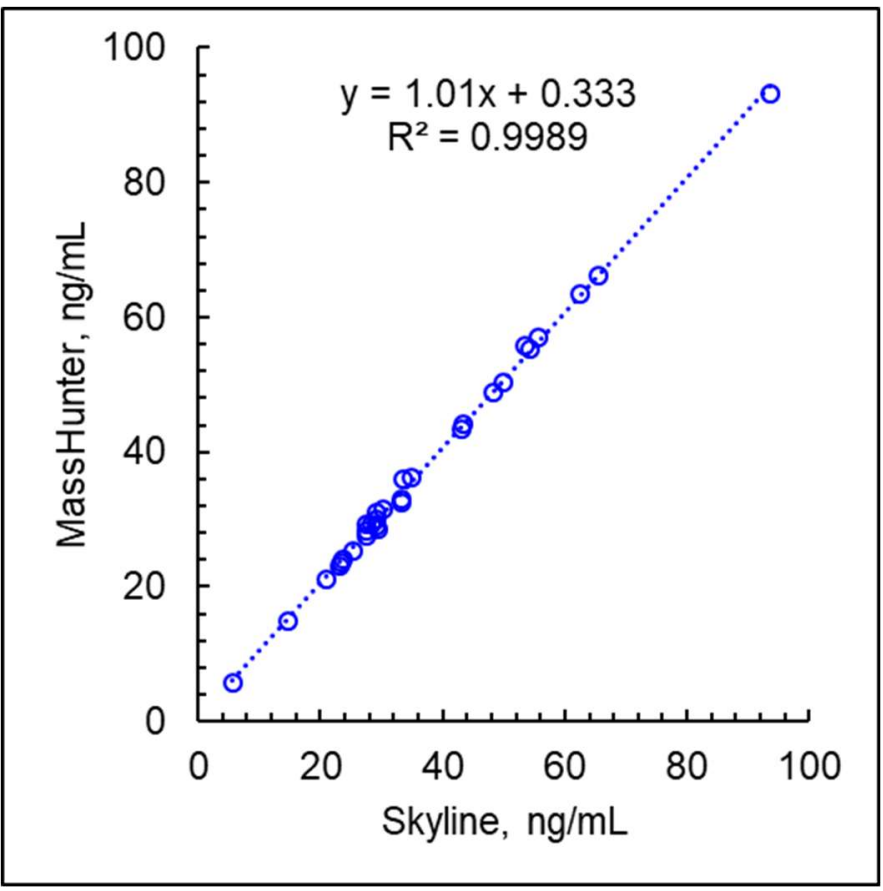

Figure S3. Correlation plot of patient samples between MassHunter and Skyline with orthogonal, equal variance regression. Good correlation is seen between the two methods.

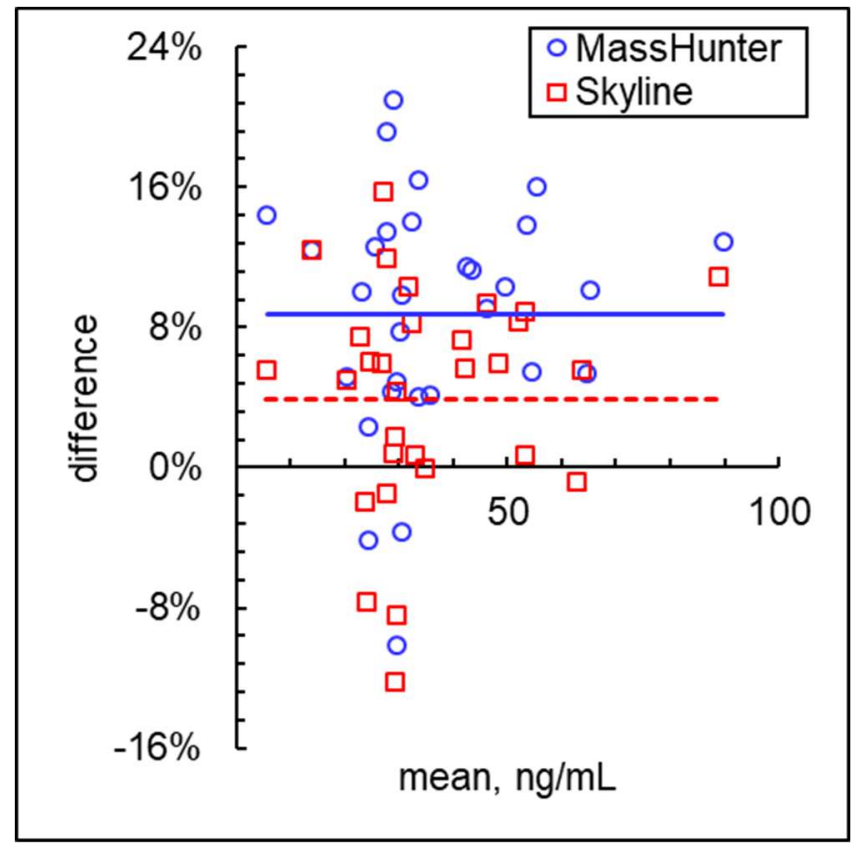

Figure S4. Difference plots for the patient sample validation run compared to the $\mathrm{LC} / \mathrm{MS} / \mathrm{MS}$ reference laboratory results using both software packages. The mean difference using skyline (red dotted line) is lower than using MassHunter (solid blue line) 


\section{Mass Hunter datafile pre-processing}

For the vendor software, the Agilent Quantitative Analysis program version B.08.00 was used for quantitation after drift time information is extracted using Agilent IM browser software version B.08.00. In the IM Browser program, the drift-time region from 28.51 to $29.85 \mathrm{~ms}$ was selected and filtered to retain retention time, mass-tocharge and intensity information. Two possible preprocessing approaches could be used for the vendor software. The drift time spectrum (e.g. Figure 3) could be used directly by summing the scans across the chromatographic peak, converting the drift time to retention time, and importing in to the quantitation software, or the chromatogram could be rebuilt by filtering only the relevant drift time window (e.g. Figure S2). Of the two, the latter approach was chosen to preserve the traditional LC/MS workflow as much as possible and because drift time is more stable than retention time from sample to sample; less variation due to time shifts is expected when a fixed drift time window is used for filtering rather than retention time window. The relative standard deviation measured across a 46 -sample experiments was $0.4 \%$ for drift time and $1.6 \%$ for retention time. The rebuilt chromatograms have lower noise than the drift-time files; by selecting a larger retention time window to capture both $25 \mathrm{OHD} 2$ and 25OHD3, a larger window is summed together than is necessary than if each analyte were selected separately. When using the chromatogram rebuilding approach, only the drift time region consistent with the open conformers is saved. Since the drift times for 25OHD2 and 25OHD3 are effectively the same, a smaller window is required than with the drift-time conversion. An "extracted drift time chromatogram" is created where only data from $28.51-29.85 \mathrm{~ms}$ is saved, creating an LC/MS datafile that 
contains only the retention time, $\mathrm{m} / \mathrm{z}$, and intensity information within that drift time region, which is compatible with the respective quantitation software package. Since drift time, as opposed to CCS, is an experimental value, dependent on the electric field, instrument geometry, gas pressure, and temperature, the drift time range is selected manually to include the two analytes and their internal standards. By quantifying using the internal standard ratio, variation in the selection window is corrected.

\section{Relative informing power of IM-HRMS}

Traditional LC-MS/MS methods that utilize a triple-quadrupole mass spectrometer, operate at unit mass resolution (resolving power $=$ ion $\mathrm{m} / \mathrm{z}$ ), whereas the LC/IM-HRMS method utilizes a high-resolution TOF mass analyzer with a resolving power over 10,000. A single stage of mass analysis affords much higher resolution of isobaric ions in addition to the separation power of IMS. The lack of fragment ions with which to improve specificity may be troublesome to a method developer, so it is useful to consider the differences in the informing power of each approach.

Efforts to quantify the informing power of tandem mass spectrometric methods include information theory as described by Fetterolf ${ }^{1}$. Peak capacity (the total number of peaks that can be observed, as used in chromatography) has been used to approximate the potential information from an IM-HRMS measurement ${ }^{2}$. For a tandem separation device, the peak capacity is the product of the resolving power of each stage and fraction of orthogonality, a measure of the difference in the properties measured. For example, LC, which separates based on affinity between a stationary and mobile phase, is orthogonal to MS, which separates based on $\mathrm{m} / \mathrm{z}$. On the other hand, CCS generally increases with $\mathrm{m} / \mathrm{z}$, reducing the fraction of orthogonality. A triple-quadrupole 
mass spectrometer with unit resolution in each stage, has a resolving power of 423 for sodiated 25OHD3 $(\mathrm{m} / \mathrm{z} 423.3)$ for the first quadrupole and 159 for the final quadrupole (based on the most common fragment ion used, $m / z$ 159.1). Assuming the fragment ion $\mathrm{m} / \mathrm{z}$ measured with the final quadrupole is $100 \%$ orthogonal to the fragment ion measured with the first, by virtue of the collision-induced dissociation, the total peak capacity is $423 \times 159 \times 1=67,000$. The IM-QTOF has a resolving power of $13,000(\mathrm{~m} / \mathrm{z}$ 423.3239) in the TOF and 52 in the IMS drift tube. Dwivedi estimates a fraction of orthogonality of $14 \%$ for drift-tube IM-HRMS ${ }^{3}$; therefore, the total peak capacity is 96,000 , slightly higher than that of the triple-quadrupole method. This is not to say that IM-MS provides better specificity that tandem MS, but that the informing power is on the same order for the two methods; i.e. nothing is lost by using IMS and high-resolution mass spectrometry.

\section{References}

(1) Fetterolf, D. D.; Yost, R. A. Added Resolution Elements for Greater Informing Power in Tandem Mass Spectrometry. Int. J. Mass Spectrom. Ion Process. 1984, 62 (1), 33-49.

(2) Lapthorn, C.; Pullen, F.; Chowdhry, B. Z. Ion Mobility Spectrometry-Mass Spectrometry (IMS-MS) of Small Molecules: Separating and Assigning Structures to lons. Mass Spectrom. Rev. 2013, 32 (1), 43-71.

(3) Dwivedi, P.; Schultz, A. J.; Hill, H. H. Metabolic Profiling of Human Blood by HighResolution Ion Mobility Mass Spectrometry (IM-MS). Int. J. Mass Spectrom. 2010, 298 (1-3), 78-90. 